\title{
Introduction to the Characterization of Hygrothermal Microcracking of Crossply Composites Reinforced by Stitched Non-woven UD Laminae
}

\author{
P.J. Liotier ${ }^{1, a}$, A. Vautrin ${ }^{1, b}$, J.M. Beraud ${ }^{2, c}$ and P. Henrat ${ }^{2, d}$ \\ ${ }^{1}$ LTDS / UMR 5513 / ECL / ENISE / ENSM-SE \\ SMS, ENSM-SE, 158, cours Fauriel - 42023 Saint-Étienne cedex 2 - France \\ ${ }^{2}$ HEXCEL Reinforcements, ZI les Nappes 38630 Les Avenières - France \\ aliotier@emse.fr, ${ }^{b}$ vautrin@emse.fr, ${ }^{c}$ jean-marc.beraud@hexcel.com, ${ }^{d}$ patrick.henrat@hexcel.com
}

Keywords: Stitched UD lamina, durability, hygrothermal cycles, microcracking.

\begin{abstract}
This article presents a first approach of hygrothermal microcracking in stitched crossply laminates. The presentation includes the definition of the material mesostructure and the selection of a characterization method adapted to the specificity of the study. The hygrothermal loading selected is discussed and the evolution of the crack density in stitched crossply composites during the loading is presented and commented.
\end{abstract}

\section{Introduction}

New high performance composites are presently being developed for advanced aerospace applications. These materials aim to minimize preconditioning to ease processing and decrease storage and raw material costs. In this study, we focus on composites reinforced by stitched nonwoven unidirectional carbon fiber laminae. These products are non-crimped, allowing taking full advantage of the high stiffness of carbon fibers, which represent the main part of the raw material cost. These kinds of reinforcements can be utilized in LCM processes - injection, infusion - aimed to lower manufacturing costs compared to prepregs (easier storage, higher throughput,...). However, the stitching introduces some new local parameters into the laminate morphology, which can induce some effects on the durability of these materials. Our objective is to study the impact of the heterogeneities due to the stitching on the behaviour of the composite during hygrothermal aging. This research is of particular interest to the aerospace industry since aircraft materials are often submitted to an ambient humidity and a wide variation of temperatures. These effects are known to induce microcracking. Hence, efficient and reliable techniques must be developed to characterize the microcracking of a complex three-dimensional material mesostructure.

\section{Materials}

This material is composed of several laminae with different orientations stitched together. The stitching has no mechanical property and is simply used to ease the handling and the processing of the reinforcement. The stitching yarns introduce ellipsoidal perturbations in the fiber alignment [1]. These perturbations have different size and shape according to the yarn size, the stitching type and the orientations of the plies. Nevertheless, these elliptical perturbations have some interest since they serve as channels to facilitate resin flow during resin infusion [2]. However, after processing, these perturbations become resin rich inclusions in the composite. In most cases, the final laminate is composed of several layers of stitched plies. For example, the stitching process allows assembling several plies into stitched stacks of two to four plies per stack. So, a laminate with twelve plies will be composed of three to six stacks, each having a different resin inclusion distribution. Moreover, the stitched plies are not oriented in the same way. The ellipsoidal inclusion rotates with the fiber orientation [3]. So it becomes a periodic three-dimensional inclusion. The spatial period is known 
for each individual stack, but in an assembly it becomes impossible to control the relative interstack offsets.

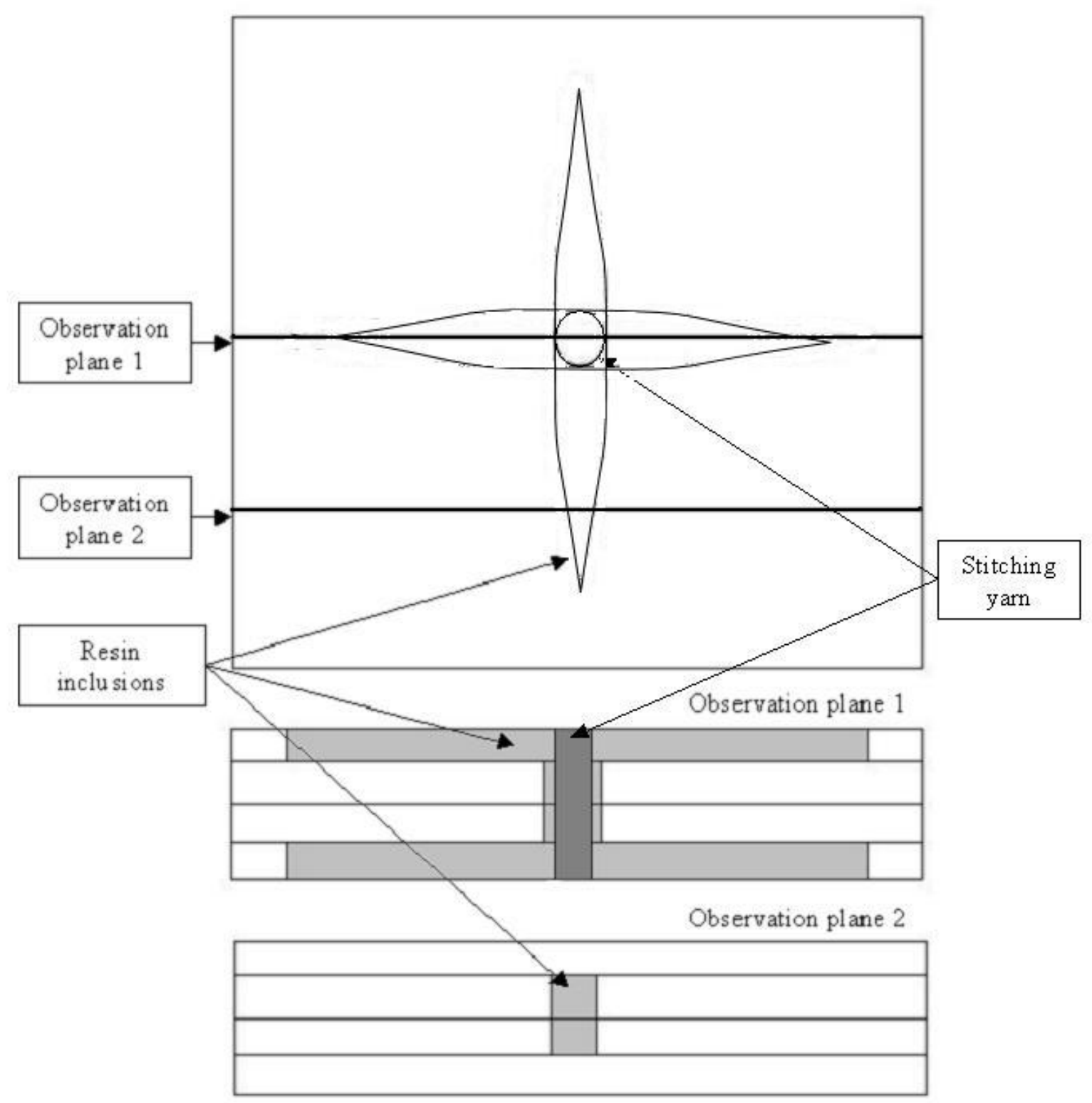

Figure 1 : Stitching area model in a biaxial $[0 / 90]_{\mathrm{S}}$ laminate

\section{Analysis method}

To study and characterize the stitching area, we select optical micrography since it is the only direct method to look inside the material [4]. Most of the studies on composite cracking limit their observation to the edge of the specimen [4-7]. In this study due to the presence of stitching, the specimen edges are not representative of what occurs inside the material. Hence, it is necessary to observe the specimen in other locations, which means that a careful analysis is required to select relevant areas. To solve this problem, we have two possibilities, we can cut the specimen in order to force the stitching to be on the edge of the sample or we can investigate internal stitching yarns. In the first approach, the results could not be representative because the stress field will be perturbed at the free edge. This approach requires identical stress fields for all specimens, which implies a perfect control of the edge position with respect to the resin inclusions.

To avoid these problems, we have selected the observation of the internal stitching yarn. A method has been developed to control the location of the observation plane. 
First we established a protocol to achieve an adequate surface state for optical micrography measurements with minimum time. Then we cut the sample at a distance equal to or bigger than the region consumed by the abrasion process. The cut is performed with a circular $\mathrm{SiC}$ blade. The cut is also done by abrasion as to not disturb the cracks. During polishing, we estimate the volume consumed by the process and we continue to polish the surface until we reach the observation plane. This method allows for an accuracy of plane localization up to $20 \mu \mathrm{m}$ and a surface status suitable for optical observations (figure 2).

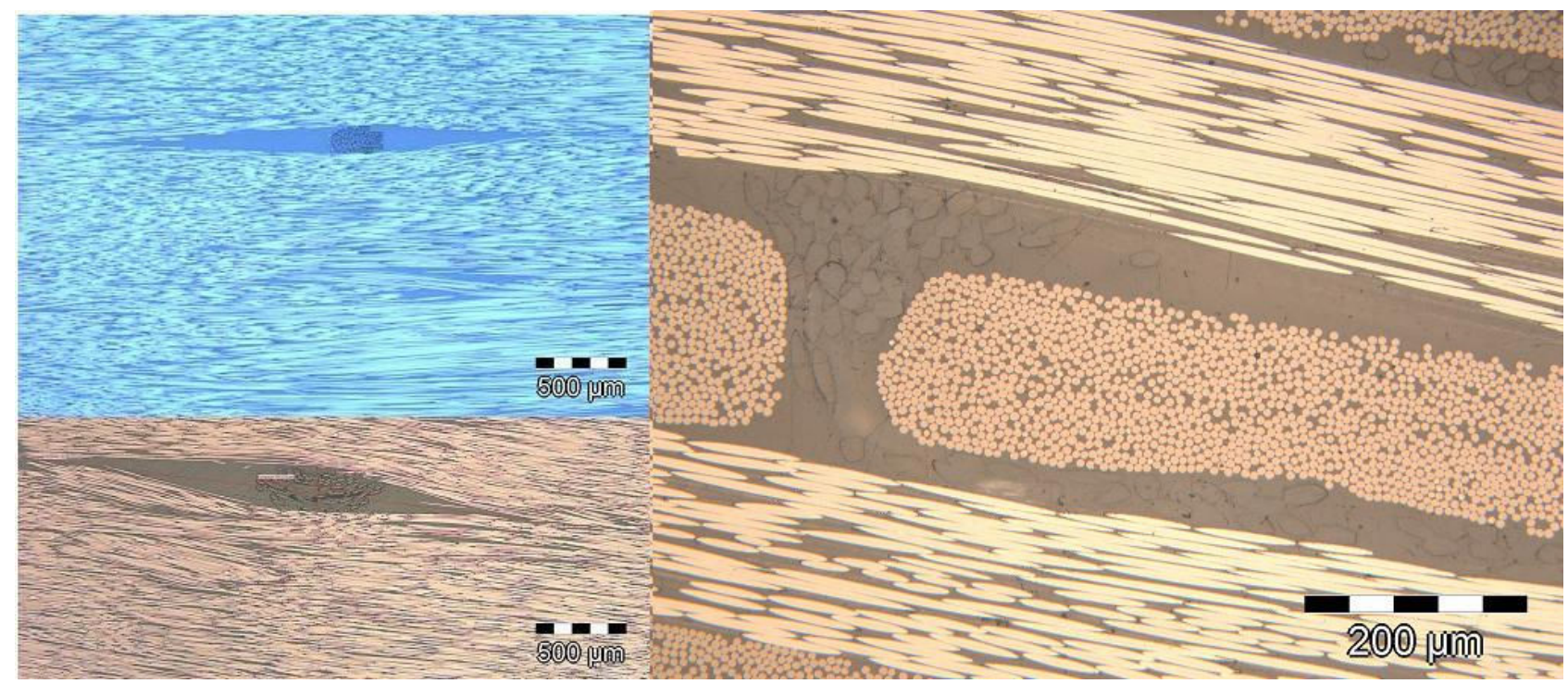

Figure 2 : Stitching micrography

\section{Hygrothermal loading}

The aging of such materials has not been yet studied. We managed to study a damaging loading which could be related to extreme value of service conditions. The temperature $-55^{\circ} \mathrm{C}$ is considered as representative of the low temperature during a subsonic flight. Such low temperature must be included in the loading because it is at low temperature that residual stresses are the highest. To ease the modeling of the sample state, we decide to split the humidification period and the thermal cycles instead of managing humidity cycles which induce transient humidity profile at each cycle. We selected for this study the hygrothermal loading defined by Klug [8]. This aging loading, presented in figure 3, is divided in 5 blocks with a humidification period at $50^{\circ} \mathrm{C}$ and $95 \% \mathrm{RH}$ and 400 thermal cycles of an hour between $80^{\circ} \mathrm{C}$ and $-55^{\circ} \mathrm{C}$ with a 15 min step at these two temperatures. Five samples of the same material are placed at the beginning of the cycles. Between each blocks, we take one sample for the analysis of several stitching lines. We are able to follow the evolution of the microcracking state near the stitching with the method previously described. To quantify the microcracking, we define a parameter, $d$, characterizing the crack density of the laminate, suited to our protocol :

$$
d=\frac{\sum_{i} N_{i}}{L \times p},
$$

where $\mathrm{N}_{\mathrm{i}}$ is the total number of cracks in ply $\mathrm{i}$ [9], $\mathrm{L}$ is the sample length and $\mathrm{p}$ is the number of plies observable in the considerate observation plane.

As mentioned previously, the stitching will create inclusions of hydrophilic resin; hence the stitching may influence the water concentration profile of a specimen. The inclusion could be a preferential diffusion path for the transverse direction. In addition it is possible for the residual stress field in the laminate to be perturbed by the inclusions, which are unreinforced areas. 


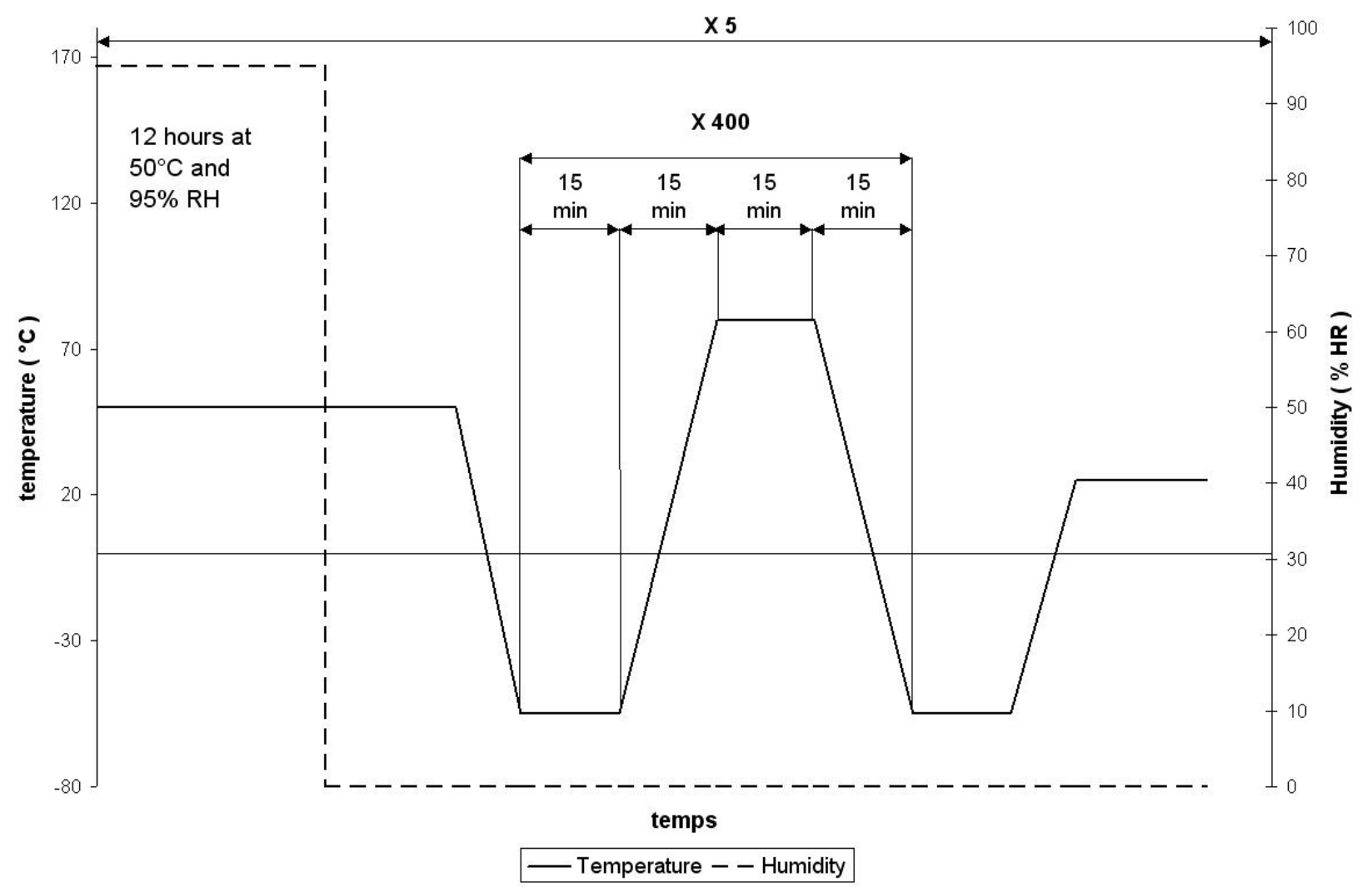

Figure 3 : Hygrothermal loading

\section{Previous results on unstitched materials}

To the author's knowledge, there are only a few papers treating the detection of microcracks on composites after pure hygrothermal solicitation and no heavy chemical modification (e.g. oxidation or modification by irradiation). Reynolds et al. [9] for instance present an accelerated aging cycle with two dry phases at $163^{\circ} \mathrm{C}$ and $-54^{\circ} \mathrm{C}$ and a wet phase at $87^{\circ} \mathrm{C}$ and $85 \% \mathrm{RH}$ for a total cycle duration of $90 \mathrm{~min}$. The authors characterized the microcracking state every 500 cycles and concluded that there is no crack before 2000 cycles on prepreg carbon/epoxy based biaxial $[0 / 0 / 90 / 90 / 0 / 0 / /]$ composite. Even for higher cycle numbers, the crack density remains lower than $2 \mathrm{~cm}^{-1}$ in eight plies where cracks are visible.

\section{Measurements on stitched biaxial $[0 / 90 / /]_{\mathrm{S} 2}$ laminates}

For the first test on stitched laminate, we decided to emphasize the specific properties of this material. We managed to have very large inclusions in the tested sample. Moreover, to simplify the characterization of the cracking paths, we chose a $[90 / 0 / 0 / 90]_{\mathrm{S}}$ laminate where the stitching line is oriented at $0^{\circ}$ and the plies are linked four by four. The crack density at different loading times is shown in figure 4 . We have conditioned two series of samples, the first one has been submitted to the complete loading history and is indexed wet and the second one was only subjected to the thermal cycles and is indexed dry (figure 4).

It seems that no significant difference is detected between the crack densities of the dry and wet specimens, meaning that moisture conditioning is not so critical. However, we notice that crack density of wet specimens tends to be lower than dry specimens that can be considered as reasonable. Those results should be confirmed in the future.

The micrographies are analysed after every block of cycles. They clearly showed that the microcracks occurred in the stitching areas (figure 5), therefore the evolution of figure 4 can be regarded as characteristic of the microcracking due to stitching. However, for cycle numbers higher 
than 1600 cycles some transverse cracks can be observed within the plies (figure 5.b), those looks similar to the ones classically found in unstitched laminates.

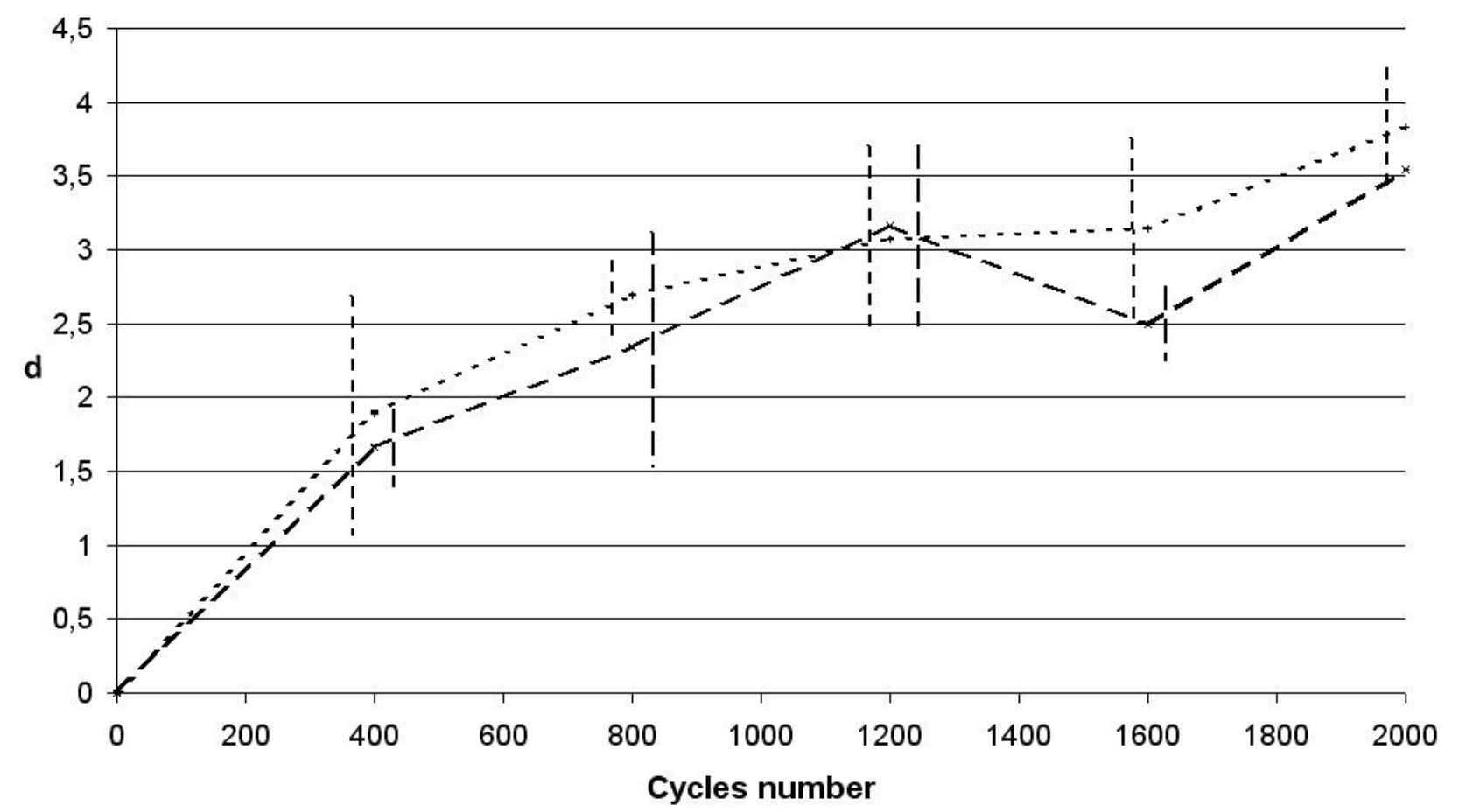

$-+-90 / 0 / 0 / 90 / /$ dry $-\div$ 90/0/0/90// wet

Figure 4 : Crack density evolution during cycles

\section{Conclusion}

This article proposes a protocol to investigate resin inclusion morphology in stitched laminate and an extension of existent methods of crack characterization due to the microcracking of stitched laminates. The principal result is that some microcracking inside the stitching areas occurs in this material. This could lead to a new path in composite microcracking analysis.

The tests with and without prehumidification period show that the influence of moisture on the crack density cycle is not significant for the material for this particular cycle.

We are now extending the study to different types of stitching yarns and stacking sequences which could have a complex effect on crack propagation. A better knowledge of crack initiation will be derived from mechanical modeling and interfaces investigation and crack propagation by X-ray microtomography imaging. 


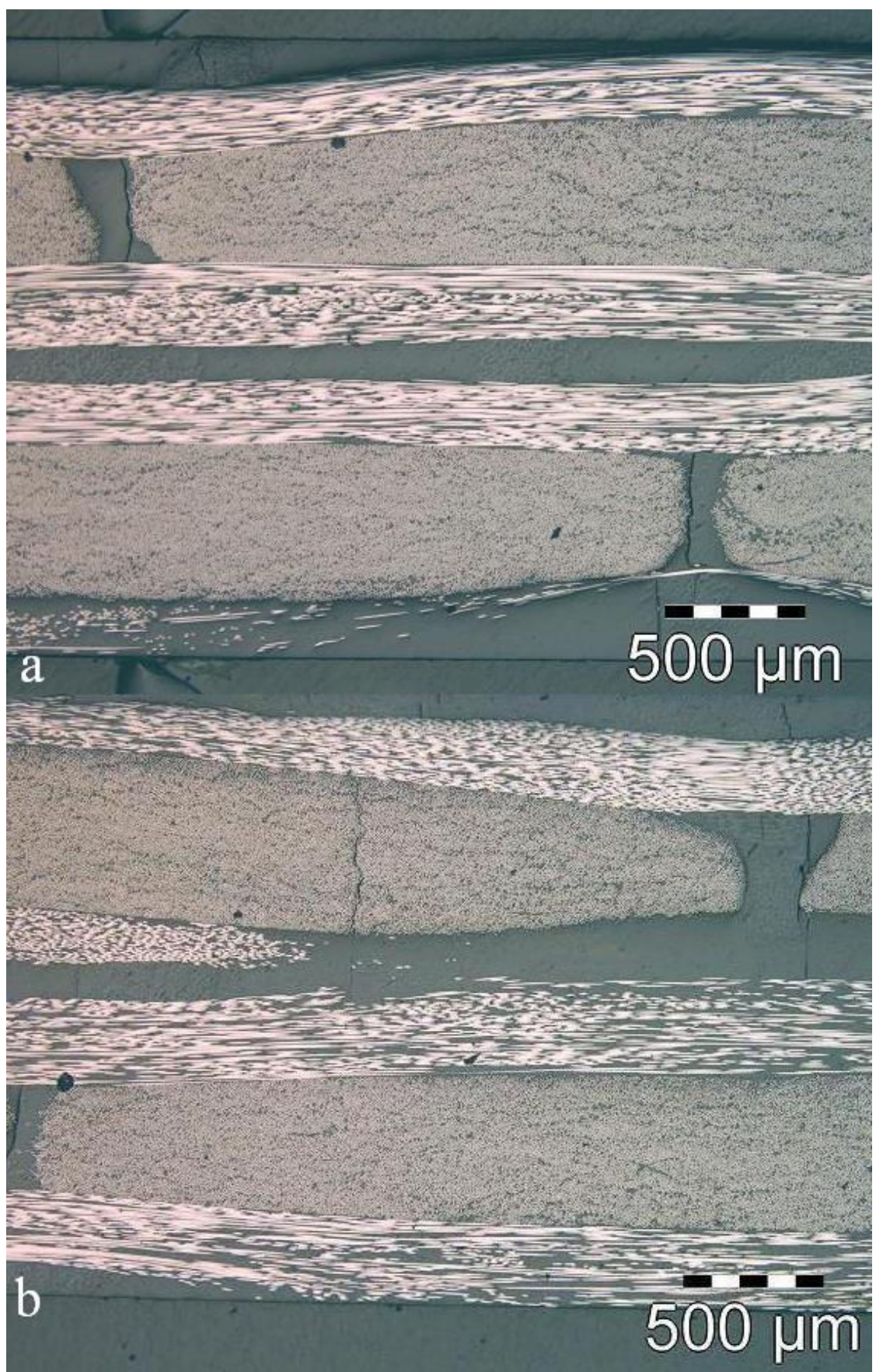

Figure 5: cracks at a) 1200 thermal cycles and 3 humidification periods and b) 2000 thermal cycles and 5 humidification periods

\section{References}

[1] S.V. Lomov et al. "Carbon composites based on multiaxial multiply stitched preforms. Part 1 : Geometry of the perform”, Composite : Part A, vol 33, 2002, p.1171-1183.

[2] S. Drapier, A. Pagot, A. Vautrin, P. Henrat, "Influence of the stitching density on transverse permeability of non-crimped new concept (NC2) multiaxial reinforcements : measurements and predictions", Composite science and tech., vol 62, 2002, p.1971-1991.

[3] Heoung-Jae Chun, Hyung-Woo Kim, Joon-Hyung Byun, "Effects of through-the-thickness stitches on the elastic behavior of multi-axial warp knit fabric composites", Composite Structures, vol. 74, 2006, p.484-494.

[4] Hayes, B.S.and Gammon L.M. , "Composites”, volume 21, ASM Handbook, 2001, p.964-972. 
[5] F. Gao et al., "Damage accumulation in woven-fabric CFRP laminates under tensile loading : Part 1. observations of damage accumulation", Composite science and tech., vol 59, 1999, p.123-136

[6] C.H. Park, H.L. McManus, "Thermally induced damage in composite laminates : predictive methodology and experimental investigation", Composite science and tech., vol 56, 1996, p.1209-1219

[7] Y.J. Weitsman, Ya-Jun Guo, "A correlation beetween fluid-induced damage and anormalous fluid sorption in polymeric composites", Composite science and tech., vol 62, 2002, p.889-908.

[8] J.H. Klug, 1999. «High-performance adhesive systems for polymer composite bonding application», Thèse de doctorat, Université de Washington.

[9] Reynolds, T.G. and McManus, H.L. “ Accelerated Tests of Environmental Degradation in Composites Materials", Composites Structures : Theory and practice, ASTM STP 1383, P. Grand and C.Q. Rousseau, Eds, American society for Testing and Materials, West Conshohocken, PA, 2000, pp.513-525.

[10] T.C. Truong et al. "Carbon composites based on multiaxial multiply stitched preforms. Part 4 : Mechanical properties of composites and damage observation", Composite : Part A, vol 36, 2005, p.1207-1221. 